\title{
Thyroiditis: can we depend on the clinical features to diagnose?
}

\author{
MB Samarawickrama ${ }^{1}$, BL Perera $^{2}$, \\ ${ }^{1}$ Lecturer, Department of Anatomy, Faculty of Medicine, University of Ruhuna, ${ }^{2}$ Consultant Surgeon, Base \\ Hospital, Gampaha.
}

e-mail address of the corresponding author,Dr.M.B. Samarawikcrama: samaramb@gmail.com

\begin{abstract}
Introduction: Thyroiditis is an inflammatory condition affecting the thyroid gland. It is a common cause for goitrous hypothyroidism. Various prevalence rates have been sited in the literature. However, the data on thyroiditis in Sri Lanka is sparse and the precise incidence in Sri Lanka is not known. The wide spread use of iodized salt is thought to be the cause for increasing incidence of thyroiditis.

Materials and Method: The data was collected from 43 patients with thyroiditis out of 350 patients with thyroid diseases who attended the surgical out patient clinic of surgical unit III of Teaching Hospital Karapitiya from July 2004 to January 2007. All these patients were studied in term of their clinical presentation, demography, clinical type of the goitre, thyroid function status, cytology of the thyroid and the mode of treatment and the data was analyzed.

Results: The total number of patients with thyroid diseases was 350 . Forty three out of the 350 patients were found to have thyroiditis. This accounts for $12 \%$ of the study group. The female to male ratio was $42: 1$. The mean age of patients with thyroiditis was 33 years. Their presenting complains were goitre 40 (93\%), voice changes $5(12 \%)$, pressure symptoms $4(9 \%)$, toxic features $4(9 \%)$, neck pain $3(7 \%)$ and enlarge cervical nodes 2(5\%) (Some patients had more than one complaint). Fifty two percent of patients had multinodular goitres. There was equal number of hypo and euthyroid patients. Only one patient was hyperthyroid. Fine Needle Aspiration Cytology (FNAC) was positive in $81 \%$ of the patients. The rest of the patients had colloid goitres and their thyroid peroxidase antibodies were elevated.

Discussion: The incidence of thyroiditis was high in this group when compared with the other studies. The commonest sub type was Hashimoto's thyroiditis which is reported to be the commonest sub type in the literature. The clinical features of these patients were not typical of thyroiditis. Therefore the clinical features did not help the diagnosis of thyroiditis in these patients. The diagnosis was mainly done by FNAC. Because of this we would like to recommend directing the investigation to find the possibility of thyroiditis in suspicious patients with thyroid diseases and further studies are required for making diagnosis and management protocols.
\end{abstract}

\section{Introduction}

Thyroiditis is an inflammatory condition affecting the thyroid gland. It is a common condition for goitrous hypothyroidism. Various prevalence rates for this condition are cited in literature ${ }^{1}$. An autopsy study in UK showed the prevalence rate of 5 to 15 percent in women and 1 to 5 percent in men. The data on thyroiditis in Sri Lanka is sparse and the precise incidence in Sri Lanka is not known ${ }^{2}$. However, the prevalence seems to be high. The widespread use of Iodized salt is thought to be the cause for this increased incidence of thyroiditis ${ }^{3}$.
Although there is no internationally accepted classification of thyroiditis ${ }^{3}$ the following sub types are mentioned.

1. Hashimoto's thyroiditis (chronic lymphocytic thyroiditis)

2. Painful thyroiditis (de Quervain's thyroiditis / subacute granulomatous thyroiditis)

3. Painless thyroiditis (subacute lymphocytic thyroiditis) 


\section{a. sporadic \\ b. postpartum}

4. Riedel's invasive fibrous thyroiditis

5. Acute suppurative thyroiditis

The natural history of the disease varies with the subtype. Inflammatory destruction of the thyroid may lead to transient thyrotoxicosis in painless sporadic thyroiditis, painless postpartum thyroiditis, and painful subacute thyroiditis. This is due to the fact that preformed thyroid hormones are released from the damaged cells of the gland. The hypothyroid phase of thyroiditis results from the gradual depletion of stored thyroid hormones. Although chronic hypothyroidism is most closely associated with Hashimoto's thyroiditis, all types of thyroiditis may progress to permanent hypothyroidism ${ }^{4}$. The most dangerous sequelae is the malignant transformation. For instance long standing lymphocytic thyroiditis may lead to lymphoma. The risk of this disease is increased by a factor of 67 in patients with Hashimoto's thyroiditis ${ }^{4}$. Therefore it is important to identify thyroiditis among thyroid disease patients.

\section{Materials and Method}

The data was collected from three hundred and fifty patients with thyroid diseases who attended the surgical out patient clinic of the surgical unit III of Teaching Hospital Karapitiya from July 2004 to January 2007. All these patients were studied in terms of their clinical presentation, demography, clinical type of the goitre, thyroid functional status, cytology of the thyroid and method of treatment.

Among these 350 patients there were 43 patients with thyroiditis. These 43 patients were studied separately to identify their clinical presentation, type of thyroid enlargement, functional status (euthyroid, hypothyroid or hyperthyroid) by mean of clinical and biochemical parameters. The following clinical parameters were used to identify the functional status. Weight loss, increased appetite, palpitation and increased sweating were taken as symptoms of hyperthyroidism while the increased pulse rate, tremors, sweaty palms and eye sings (lid lag, lid retraction and exophthalmos) were taken as signs of hyperthyroidism. Hypothyroidism was diagnosed clinically using symptoms like weight gain, lethargy, constipation and signs like facial puffiness, dry skin, slowness of movements and slow reflexes. The biochemical assessment was done by using the level of Thyroid Stimulating Hormone (TSH) and Free Thyroxin Hormone ( $\left.\mathrm{FT}_{4}\right)$ in the blood. The results of FNAC and thyroid peroxidase antibody were used to confirm the diagnosis of thyroiditis.

\section{Results}

The total number of patients with thyroiditis was 43 . This accounts for $12 \%$ of the total number of patients with thyroid diseases assessed during this period. There was only one male patient. Therefore, the female to male ratio in this study was $42: 1$. The mean age of this study group was 33 years (range 12-63 years). Table 1 shows the different symptoms they had on presentation.

Table 1 - Symptoms in patients with thyroiditis on presentation

\begin{tabular}{|l|l|}
\hline Symptom & Number \\
\hline Pressure symptoms* & 04 \\
\hline Toxic symptoms & 04 \\
\hline Pain & 03 \\
\hline Change in voice & 05 \\
\hline Goitre** & 27 \\
\hline
\end{tabular}

* Discomfort on swallowing and/or difficulty in breathing.

** These patients did not complain of any other symptom.

Forty patients had goitres while three patients had normal thyroids on examination. Fifty two percent of them had multinodular goitres (MNG) (Table 2). According to the thyroid hormonal assay, $49 \%$ of them were hypothyroid at presentation (Table 3). 
Table 2 - State of the thyroid gland on palpation

\begin{tabular}{|l|c|}
\hline State of the thyroid gland & Number of patients \\
\hline MNG & 21 \\
\hline Diffuse goitre (DG) & 10 \\
\hline Solitary thyroid nodule (STN) & 09 \\
\hline Normal thyroid & 03 \\
\hline
\end{tabular}

Table 3 - Functional status of the thyroid gland according to hormonal assays

\begin{tabular}{|l|c|}
\hline Functional state & Number of patients \\
\hline Hypothyroid & 21 \\
\hline Euthyroid & 21 \\
\hline Hyperthyroid & 01 \\
\hline
\end{tabular}

The FNAC showed cytological features of thyroiditis in $81 \%$ of the study group. The rest of the group had colloid goitres. (Table 4) The most common type of thyroiditis was the chronic lymphocytic thyroiditis or Hashimoto's thyroiditis.

\section{Table 4 - Finding of FNAC of the thyroid gland}

\begin{tabular}{|l|c|}
\hline Type of thyroiditis & Number of patients \\
\hline Chronic lymphocytic thyroiditis & 31 \\
\hline de Quervain's thyroiditis & 01 \\
\hline Subacute lymphocytic thyroiditis & 02 \\
\hline Colloid goitre* & 05 \\
\hline
\end{tabular}

* Thyroid peroxidase antibody was positive in these patients.

\section{Discussion}

Thyroiditis is a benign condition involving the thyroid gland. The inflammation may be caused by an infection (e.g. Bacteria) or formation of autoimmune antibodies against the thyroid tissue. But the cause for subtypes Riedel's invasive fibrous thyroiditis and painful thyroiditis is not known ${ }^{4}$. Depending on this and their clinical pattern there are several sub types of thyroiditis ${ }^{1}$. These include Hashimoto's thyroiditis, painful thyroiditis, painless thyroiditis (sporadic and postpartum) Riedel's invasive fibrous thyroiditis and acute suppurative thyroiditis. There were patients with Hashimoto's thyroiditis, painful thyroiditis and painless thyroiditis in this study. We did not encounter postpartum thyroiditis, Riedel's invasive fibrous thyroiditis and acute suppurative thyroiditis. It shows the rarity of these latter conditions. The commonest type is the Hashimoto's thyroiditis which was the commonest type in this series as well.

There are few aetiological factors for thyroididtis ${ }^{1,4}$. These include genetic factors, smoking, correction of iodine insufficiency, drugs (amiodarone, Lithium) and radiation. None of these patients had risk factors other than consuming iodized salt which is thought to be the main aetiological factor for increase incidence of thyroiditis. This is due to the fact that iodine induces autoimmunity in the thyroid gland ${ }^{1}$.

The clinical presentation of patients with thyroiditis may vary. They can present with hypothyroidism, goitre, or both and other symptoms like pain in the neck'. The goitre is usually diffuse and may be tender. However in this series the clinical features did not support the diagnosis of thyroiditis. Fifty percent of the patients were hypothyroid while one patient was hyperthyroid and the others were euthyroid. On the other hand forty patients had enlarged thyroid at presentation and three patients had normal thyroid. Out of these, 21 patients had multinodular goitres (MNG). Therefore, the commonest type of goitre was MNG rather than diffuse goitres which were encountered only in $23 \%$ of the study group. None of these patients' goitres were tender on palpation. Another study done on a group of Sri Lankan patients with chronic lymphocytic thyroiditis also reported that their clinical presentation differ from the symptoms cited in the literature ${ }^{2}$.

When the clinical presentations give no clue to diagnosis investigations are very useful. The FNAC was positive for thyroiditis in $81 \%$ of the patients. The remaining 19\% had colloid goitre on FNAC findings. In this latter group diagnosis was made by the presence of positive thyroid peroxidase antibodies. Thyroid peroxidase antibody was positive in $50 \%$ of the patients. Therefore, the diagnosis mainly depended on FNAC. The clinical subtypes were identified according to FNAC findings.

Similarly their functional status was also confirmed with biochemical parameters $\left(\mathrm{TSH}, \mathrm{T}_{4}\right.$ ) rather than clinical presentations. However there was one 
hyperthyroid patient and he had symptoms of hyperthyroidism. But not all the hypothyroid patients (21/43) had typical features of hypothyroidism. In fact some of them were clinically euthyroid even though biochemically hypothyroid (High TSH and low $\mathrm{T}_{4}$ ). The hypothyroid phase of thyroiditis results from the gradual depletion of stored thyroid hormones. Although chronic hypothyroidism is most closely associated with Hashimoto's thyroiditis, all types of thyroiditis may progress to permanent hypothyroidism ${ }^{4}$. If it is not treated, hypothyroidism can lead to other health problems like goitre, increase risk of heart diseases, depression, and congenital defects if mother is affected. Therefore, it is important to identify the patients with thyroiditis early. This may enable doctors to treat them adequately and prevent them from becoming hypothyroid. However, this study shows the difficulty in identifying them on clinical grounds. Therefore, we would like to recommend directing the investigations to find the possibility of thyroiditis in suspicious patients with thyroid diseases. When considering the investigations both FNAC and thyroid peroxidase antibodies have to be done to avoid missing patients with thyroiditis.

\section{References}

1. Colin M Dayan, Gilbert H Daniels. Chronic Autoimmune Thyroiditis. New Eng J of Med July 1996; 335(2): 99-107.

2. Fernando R, Coomaraswamy W, Sathesan B. Clinical features of chronic lymphocytic thyroiditis are they different in our patients. The Annual Scientific Session of the College of Surgeon of Sri Lanka. $18^{\text {th }}-20^{\text {th }}$ Aug 2005.

3. LDKE Premawardhana, AB Parkes, G Mazziotti, JH Lazarus. Autoimmune thyroiditis after elimination of iodine deficiency in Sri Lanka. Thyroid December 1, 2003; 13(12): 1187-1188.

4. N Pearce, Alan P Farwell, Lewis E Braverman. Thyroiditis Elizabeth. New Eng Jof Med June 2003; 348(26): 2646-55. 